\title{
Perfil de dispensação de opioides no Brasil entre os anos de 2014 e 2018
}

\author{
Analysis of dispensation of opioids in Brazil: 2014-2018 \\ Perfil de dispensación de opioides en Brasil entre 2014 y 2018
}

\section{Resumo}

Foi objetivo do estudo descrever o perfil de dispensação de opioides no Brasil entre os anos de 2014 e 2018 . Trata-se de um estudo longitudinal e retrospectivo que coletou dados da dispensação de opioides por farmácias, drogarias e hospitais, entre janeiro de 2014 e dezembro de 2018, a partir do Sistema Nacional de Gerenciamento de Produtos Controlados (SNGPC), gerenciado pela Agência Nacional de Vigilância Sanitária (ANVISA). As variáveis analisadas foram: número de opioides dispensados; estado da dispensação e origem do registro profissional. Um total de 60.675.488 de medicamentos foi comercializado, sendo o fosfato de codeína o princípio ativo mais dispensado, seguido do cloridrato de tramadol, que juntos representaram 95,9\% (2014), 96,6\% (2015), 96,2\% (2016), 95,4\% (2017) e 95,7\% (2018) das prescrições legais. Em praticamente todos os anos, a média de medicamentos vendidos se manteve superior a 5.000 unidades por cada 100.000 habitantes. São Paulo foi o estado que mais comercializou opioides. Quanto à origem do registro profissional do prescritor, médicos responderam por 93,2\% da medicação dispensada. Dentre os opioides dispensados, o fosfato de codeína foi o mais prescrito e não foi verificada tendência de aumento na dispensação no período avaliado.

Palavras-chave: Psicotrópicos; Analgésicos opioides; Prescrições de medicamentos; Dor; Entorpecentes.

\begin{abstract}
The aim of this study was to describe the opioid dispensing profile in Brazil between 2014 and 2018. Data were collected on the number and type of opioid dispensed after professional prescription, between January 2014 and December 2018, from the National Controlled Products Management System (SNGPC), managed by the National Health Surveillance Agency (ANVISA), having as object of study the analysis of the variables: (1) Active Principle; (2) Quantity dispensed by state and (3) Origin of Professional Registration. A total of 60.675.488 drugs were marketed, with codeine phosphate being the most widely dispensed active ingredient, followed by tramadol hydrochloride, which together accounted for 95.9\% (2014), 96.6\% (2015), 96.2\% (2016), 95.4\% (2017) and 95.7\% (2018). In practically every year, the average of medicines sold remained above 5,000 units per 100,000 inhabitants. São Paulo was the state that most commercialized opioids, and regarding the origin of the prescriber's professional record, doctors accounted for $93.2 \%$ of the medication dispensed. It was concluded that codeine phosphate was the most prescribed among the opioids dispensed. There was no trend towards an increase in dispensing in the period evaluated.
\end{abstract}

Keywords: Psychotropic drugs; Analgesics, opioid; Drug prescriptions; Pain; Narcotics. 


\begin{abstract}
Resumen
O objetivo presente do estudo foi describir el perfil de dispensación de opioides en Brasil entre 2014 y 2018. Se trata de un estudio longitudinal y retrospectivo que recopiló datos sobre dispensación de opioides por farmacias, droguerías y hospitales, entre enero de 2014 y diciembre de 2018, de los Productos Controlados Nacionales. Sistema de Gestión (SNGPC), gestionado por la Agencia Nacional de Vigilancia Sanitaria (ANVISA). Las variables analizadas fueron: número de opioides dispensados; estado de dispensa y origen del registro profesional. Se comercializaron un total de 60.675.488 medicamentos, siendo el fosfato de codeína el ingrediente activo más dispensado, seguido del clorhidrato de tramadol, que en conjunto representaron $95,9 \%$ (2014), 96,6\% (2015), 96,2\% (2016), 95,4\% (2017) у 95,7\% (2018) de los requisitos legales. Prácticamente todos los años, el promedio de venta de medicamentos se mantuvo por encima de las 5.000 unidades por cada 100.000 habitantes. São Paulo fue el estado que vendió más opioides. En cuanto al origen del historial profesional del prescriptor, los médicos concentraron el 93,2\% de los medicamentos dispensados. Entre los opioides dispensados, el fosfato de codeína fue el más prescrito y no hubo tendencia al aumento de dispensación en el período evaluado.
\end{abstract}

Palabras clave: Psicotrópicos; Analgésicos opioides; Prescripciones de medicamentos; Dolor; Narcóticos.

\title{
1. Introdução
}

A prescrição de medicamentos é uma das estratégias de saúde mais utilizadas nas mais diversas práticas clínicas e o uso adequado dos medicamentos é um processo complexo e dependente de diretrizes farmacotécnicas apropriadas à condição clínica individual de cada paciente (Brasil, 2006).

O uso racional de medicamentos é uma recomendação da Organização Mundial da Saúde (OMS), em âmbito mundial, e mais recentemente do Ministério da Saúde no Brasil, que tem tomado diferentes medidas restritivas e realizado campanhas para o uso racional de medicamentos compreendido quando os pacientes recebem medicamentos eficazes e seguros para as suas condições clínicas em doses adequadas às suas necessidades individuais, por um período adequado e ao menor custo para si e para a comunidade (Brasil, 2012).

A contínua prescrição e o uso indiscriminado de medicações, bem como o hábito da automedicação, podem levar ao abuso ou mesmo à dependência química. Os analgésicos opioides estão entre as medicações normalmente utilizadas para o tratamento da dor aguda e crônica, contudo, o grande risco no uso prolongado dessas medicações propicia o aparecimento de sua principal complicação, o vício (Volkow et al., 2011; Nascimento \& Sakata, 2011). Alguns fatores que contribuem para o aumento do uso de opioides nas práticas em saúde, incluem a prescrição excessiva de analgésicos legalizados e o fato de serem excelentes medicações para o tratamento da dor crônica aumentando o manejo dessas drogas durante as práticas clínicas (Meyer et al., 2013).

Estudo conduzido em uma população de trabalhadores de diversas áreas na França encontrou taxas de dependência e de uso de medicações psicoativas nas faixas de 3,5\% e 9,1\%, dos quais 2,1\% referiram uso de analgésicos opioides (NgoundoMbongue et al., 2005).

A ocorrência de mortes relacionadas ao consumo desenfreado de opioides é uma constante em países desenvolvidos. Nos Estados Unidos (Rudd et al., 2016; Gomes et al., 2018; Priest et al., 2020), reportou-se que em um período de três anos (2014-2017) o número de mortes pelo uso desse tipo de medicação aumentou 66\%, passando de 28.647 para 47.600 , sendo esse aumento principalmente verificado em pacientes que buscaram tratamento por abuso de heroína, dos quais $75 \%$ referiram o vício em opioides como consequência do tratamento da dependência química da droga.

No ano de 2016, uma média de 9.000 mortes foi registrada em virtude de overdose provocada pelo uso de opioides, segundo dados coletados em países da União Europeia, Turquia e Noruega. No mesmo ano, os Estados Unidos passaram pelo que foi denominado de Crise do Opioide, quando a overdose matou cerca de 63.632 pessoas, dos quais $66 \%$ tiveram acesso as medicações a partir de prescrições médicas ou de fontes ilícitas (Meyer et al., 2013).

Apesar de apresentar poucos dados referidos em literatura, o consumo desse tipo de medicação no Brasil vem ganhando força. A partir de dados extraídos do Sistema Nacional de Gerenciamento de Produtos Controlados (SNGPC), foi 
referido que entre os anos de 2009 e 2015 a prescrição de opioides no Brasil saltou de 1.601.043 para 9.045.945, representando um aumento de $465 \%$ em um espaço de seis anos, sendo os produtos à base de codeína os que mais contribuíram para esse aumento (Krawczyk et al., 2018).

O consumo ainda é baixo quando comparados com a totalidade da população brasileira. Segundo Calônego (2020), a existência do receio médico seja pela falta de conhecimento da farmacologia ou da legislação normativas desses medicamentos; bem como o medo exagerado por parte do paciente em desenvolver o vício e a dependência com o uso dessas medicações são entraves considerados para dificultar a correta prescrição de opioides.

No Brasil, a prescrição de opioides pode ser realizada por três classes profissionais: médicos, dentistas e veterinários e segue critérios estabelecidos pela legislação pertinente, com a obrigatoriedade da retenção de receita especial. Até o presente momento, não existe referência acerca da prescrição de opioides quanto à classe profissional, e por estados brasileiros. Diante disso o presente estudo descreveu os opioides dispensados no Brasil, entre os anos de 2014 e 2018 e as diferentes classes profissionais habilitadas a realizar a indicação terapêutica dos opioides.

\section{Metodologia}

\section{Tipo e local do estudo}

Foi realizado um estudo observacional, longitudinal e retrospectivo, de base nacional, considerando dados sobre número e tipo de prescrições de opioides comercializadas legalmente no Brasil (após apresentação de receita médica) por meio de dados coletados do Sistema Nacional de Gerenciamento de Produtos Controlados (SNGPC), ferramenta criada em 2007 a partir da RDC No 27, de 30 de março de 2007 e gerenciada pela Agência Nacional de Vigilância Sanitária (ANVISA), que monitora as movimentações de entrada e saída de medicamentos dispensados em farmácias, drogarias e hospitais no Brasil, pela escrituração obrigatoriamente eletrônica.

\section{Coleta dos dados}

A coleta dos dados foi realizada no SNGPC entre o período de 01 de janeiro de 2014 a 31 de dezembro de 2018, considerando-se as seguintes variáveis:

(1) princípio ativo: produtos com codeína (codeína e fosfato de codeína), fentanil (citrato de sufentanila, cloridrato de remifentanila e fentanila), oxicodona (cloridrato de oxicodona) e tramadol (cloridrato de tramadol), prescritos individualmente ou em associações entre si e/ou com outras medicações;

(2) quantidade dispensada por estado brasileiro e,

(3) registro profissional do prescritor (Conselho Regional de Medicina - CRM; Médicos Intercambistas com Registro do Ministério da Saúde (RMS), Conselho Regional de Odontologia - CRO e Conselho Regional de Medicina Veterinária CRMV).

\section{Análise dos dados}

A análise foi realizada por meio de estatística descritiva, sendo apresentado em tabelas de distribuição de frequências absolutas e relativas. Calculou-se as porcentagens acumuladas de produtos comercializados por ano e por órgão de registro do profissional. Foram também analisadas as quantidades comercializadas por 100 mil habitantes, onde foram consideradas as contagens da população realizadas pelo Instituto Brasileiro de Geografia e Estatística (IBGE-CENSO) nos anos avaliados. As análises foram realizadas com auxílio do software estatístico R Core Team. 


\section{Procedimentos éticos}

Este estudo foi conduzido de acordo com os preceitos determinados pela Resolução 466/12 para estudo com seres humanos, da Comissão Nacional de Ética em Pesquisa (CONEP) do Conselho Nacional de Saúde, submetido à apreciação do Comitê de Ética institucional, sendo dispensado da apreciação, sob o parecer n 3.267.495.

\section{Resultados}

Foram comercializados no período um total de 60.675 .488 medicamentos sendo possível observar que o fosfato de codeína foi o princípio ativo mais dispensado, representando 55,3\%, 58,6\%, 60,0\%, 49,4\% e 55,6\% dos opioides prescritos nos anos de 2014 a 2018 respectivamente. O cloridrato de tramadol foi o segundo mais comercializado e juntos, esses medicamentos representaram quase que a totalidade dos opioides prescritos no período, perfazendo totalidades de 95,9\%, $96,6 \%, 96,2 \%, 95,4 \%$ e $95,7 \%$ nos respectivos anos (Tabela 1 ).

Tabela 1. Porcentagem acumulada de opioides dispensados em farmácias, drogarias e hospitais no Brasil em função do princípio ativo e do ano de estudo, segundo o Sistema Nacional de Gerenciamento de Produtos Controlados (SNGPC), com destaque para os quatros principais comercializados. (Brasil, anos de 2014 a 2018).

\begin{tabular}{|c|c|c|c|}
\hline Ano & Princípio ativo & $\begin{array}{l}\text { Quantidade do produto } \\
\text { comercializada }\end{array}$ & $\begin{array}{c}\text { Porcentagem } \\
\text { acumulada }\end{array}$ \\
\hline \multirow[t]{5}{*}{2014} & Fosfato de codeína & 6.135 .544 & 55,3 \\
\hline & Cloridrato de tramadol & 4.494 .011 & 95,9 \\
\hline & Sulfato de morfina pentaidratada & 155.427 & 97,3 \\
\hline & Cloridrato de oxicodona & 149.667 & 98,6 \\
\hline & Outros Opioides & 154.466 & 100 \\
\hline Total & & 11.089 .115 & \\
\hline \multirow[t]{5}{*}{2015} & Fosfato de codeína & 8.862 .221 & 58,6 \\
\hline & Cloridrato de tramadol & 5.752 .696 & 96,6 \\
\hline & Sulfato de morfina pentaidratada & 178.388 & 97,8 \\
\hline & Cloridrato de oxicodona & 164.003 & 98,9 \\
\hline & Outros Opioides & 171.268 & 100 \\
\hline 2015 Total & & 15.128 .576 & \\
\hline \multirow[t]{5}{*}{2016} & Fosfato de codeína & 3.799 .658 & 60,0 \\
\hline & Cloridrato de tramadol & 2.289 .888 & 96,2 \\
\hline & Cloridrato de metadona & 95.490 & 97,7 \\
\hline & Sulfato de morfina pentaidratada & 67.047 & 98,8 \\
\hline & Outros Opioides & 76.890 & 100 \\
\hline 2016 Total & & 6.328 .973 & \\
\hline \multirow[t]{5}{*}{2017} & Fosfato de codeína & 7.318 .639 & 49,4 \\
\hline & Cloridrato de tramadol & 6.802 .920 & 95,4 \\
\hline & Sulfato de morfina pentaidratada & 230.171 & 96,9 \\
\hline & Cloridrato de metadona & 196.644 & 98,2 \\
\hline & Outros Opioides & 259.883 & 100 \\
\hline Total & & 14.808 .257 & \\
\hline \multirow[t]{5}{*}{2018} & Fosfato de codeína & 7.412 .616 & 55,6 \\
\hline & Cloridrato de tramadol & 5.332 .218 & 95,7 \\
\hline & Sulfato de morfina pentaidratada & 191.383 & 97,1 \\
\hline & Cloridrato de metadona & 174.241 & 98,4 \\
\hline & Outros Opioides & 210.109 & 100 \\
\hline Total & & 13.320 .567 & \\
\hline Total Geral & & 60.675 .488 & \\
\hline
\end{tabular}

Fonte: Elaboração própria dos autores (2020)

A venda de opioides variou entre 11.089.115 unidades no ano de 2014 e 13.320 .567 unidades no ano de 2018. As médias de medicamentos vendidos em cada ano se mantiveram superiores a 5.000 unidades por cada 100.000 habitantes, com 
exceção do ano de 2016, onde foi evidenciada uma queda significativa na quantidade de opioides vendidos no país, sendo contabilizada média equivalente a 3.000 unidades por cada 100.000 habitantes (Gráfico 1).

Gráfico 1. Quantidade de opioides comercializados em farmácias, drogarias e hospitais no Brasil a cada 100.000 habitantes em função do ano, segundo o Sistema Nacional de Gerenciamento de Produtos Controlados (SNGPC). (Brasil, anos de 2014 a 2018).

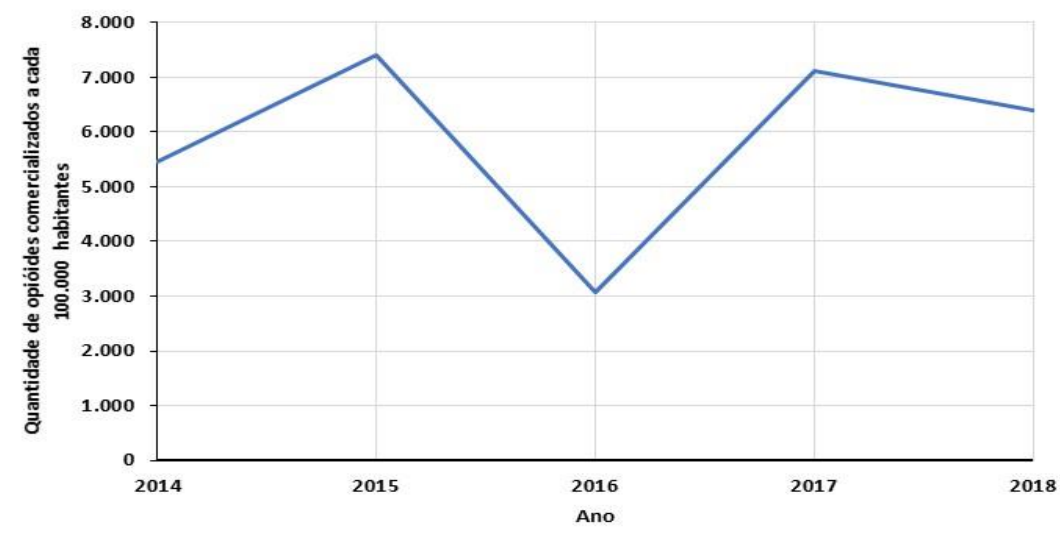

Fonte: Elaboração própria dos autores (2020).

Ao se comparar a frequência relativa de opioides comercializados por ano, entre os estados brasileiros (Tabela 2), observa-se que estado de São Paulo foi o estado que mais comercializou em praticamente todos os anos, representando 27,1\%, $21,8 \%, 31,0 \%, 25,0 \%$ e $23,5 \%$ do total comercializado de 2014 a 2018, com exceção ao ano de 2015 cujo estado que mais comercializou opioide foi o Rio Grande do Sul com $23,0 \%$ da totalidade dispensada no ano contra $21,8 \%$ da totalidade dispensada pelo estado de São Paulo. No total, os estados que mais comercializaram nos quatro anos de estudo foram São Paulo (24,9\%), Minas Gerais (12,5\%), Rio Grande do Sul (12,3\%), Paraná (10,5\%), Goiás (5,7\%), Rio de Janeiro (5,5\%) e Bahia $(4,7 \%)$. 
Tabela 2. Prescrição de opioides dispensados em farmácias, drogarias e hospitais por estado brasileiro, segundo o Sistema Nacional de Gerenciamento de Produtos Controlados (SNGPC) (Brasil, anos de 2014 a 2018).

\begin{tabular}{|c|c|c|c|c|c|c|}
\hline \multirow{3}{*}{ Estado de registro do profissional } & \multicolumn{6}{|c|}{ Ano } \\
\hline & 2014 & 2015 & 2016 & 2017 & 2018 & Total \\
\hline & $\mathrm{N}(\%)$ & $\mathrm{N}(\%)$ & $\mathrm{N}(\%)$ & $\mathrm{N}(\%)$ & $\mathrm{N}(\%)$ & $\mathrm{N}(\%)$ \\
\hline Acre & $11476(0,1 \%)$ & $\begin{array}{r}14392 \\
(0,1 \%)\end{array}$ & $6713(0,1 \%)$ & $\begin{array}{r}18373 \\
(0,1 \%)\end{array}$ & $\begin{array}{r}18468 \\
(0,1 \%)\end{array}$ & $\begin{array}{r}69422 \\
(0,1 \%)\end{array}$ \\
\hline Alagoas & $73880(0,7 \%)$ & $\begin{array}{r}89859 \\
(0,6 \%)\end{array}$ & $\begin{array}{r}80768 \\
(1,3 \%)\end{array}$ & $\begin{array}{r}111641 \\
(0,8 \%)\end{array}$ & $\begin{array}{r}98427 \\
(0,7 \%)\end{array}$ & $\begin{array}{r}454575 \\
(0,7 \%)\end{array}$ \\
\hline Amazonas & $27318(0,2 \%)$ & $\begin{array}{r}35333 \\
(0,2 \%)\end{array}$ & $\begin{array}{r}18488 \\
(0,3 \%)\end{array}$ & $\begin{array}{r}40230 \\
(0,3 \%)\end{array}$ & $\begin{array}{r}35847 \\
(0,3 \%)\end{array}$ & $\begin{array}{r}157216 \\
(0,3 \%)\end{array}$ \\
\hline Amapá & $9394(0,1 \%)$ & $\begin{array}{r}11003 \\
(0,1 \%)\end{array}$ & $4793(0,1 \%)$ & $\begin{array}{r}10832 \\
(0,1 \%)\end{array}$ & $8666(0,1 \%)$ & $\begin{array}{r}44688 \\
(0,1 \%)\end{array}$ \\
\hline Bahia & $\begin{array}{r}478796 \\
(4,3 \%)\end{array}$ & $\begin{array}{r}967317 \\
(6,4 \%)\end{array}$ & $\begin{array}{r}212099 \\
(3,4 \%)\end{array}$ & $\begin{array}{r}636066 \\
(4,3 \%)\end{array}$ & $\begin{array}{r}532159 \\
(4,0 \%)\end{array}$ & $\begin{array}{r}2826437 \\
(4,7 \%)\end{array}$ \\
\hline Ceará & $\begin{array}{r}213295 \\
(1,9 \%)\end{array}$ & $\begin{array}{r}618741 \\
(4,1 \%)\end{array}$ & $\begin{array}{r}95078 \\
(1,5 \%)\end{array}$ & $\begin{array}{r}345033 \\
(2,3 \%)\end{array}$ & $\begin{array}{r}239799 \\
(1,8 \%)\end{array}$ & $\begin{array}{r}1511946 \\
(2,5 \%)\end{array}$ \\
\hline Distrito Federal & $\begin{array}{r}185771 \\
(1,7 \%)\end{array}$ & $\begin{array}{r}210312 \\
(1,4 \%)\end{array}$ & $\begin{array}{r}77469 \\
(1,2 \%)\end{array}$ & $\begin{array}{r}402614 \\
(2,7 \%)\end{array}$ & $\begin{array}{r}214774 \\
(1,6 \%)\end{array}$ & $\begin{array}{r}1090940 \\
(1,8 \%)\end{array}$ \\
\hline Espírito Santo & $\begin{array}{r}198887 \\
(1,8 \%)\end{array}$ & $\begin{array}{r}221742 \\
(1,5 \%)\end{array}$ & $\begin{array}{r}114405 \\
(1,8 \%)\end{array}$ & $\begin{array}{r}242769 \\
(1,6 \%)\end{array}$ & $\begin{array}{r}203362 \\
(1,5 \%)\end{array}$ & $\begin{array}{r}981165 \\
(1,6 \%)\end{array}$ \\
\hline Goiás & $\begin{array}{r}738117 \\
(6,7 \%)\end{array}$ & $\begin{array}{r}517825 \\
(3,4 \%)\end{array}$ & $\begin{array}{r}256907 \\
(4,1 \%)\end{array}$ & $\begin{array}{r}620514 \\
(4,2 \%)\end{array}$ & $\begin{array}{r}1267642 \\
(9,5 \%)\end{array}$ & $\begin{array}{r}3401005 \\
(5,6 \%)\end{array}$ \\
\hline Maranhão & $87847(0,8 \%)$ & $\begin{array}{r}108546 \\
(0,7 \%)\end{array}$ & $\begin{array}{r}84486 \\
(1,3 \%)\end{array}$ & $\begin{array}{r}118819 \\
(0,8 \%)\end{array}$ & $\begin{array}{r}100573 \\
(0,8 \%)\end{array}$ & $\begin{array}{r}500271 \\
(0,8 \%)\end{array}$ \\
\hline Minas Gerais & $\begin{array}{r}1510217 \\
(13,6 \%)\end{array}$ & $\begin{array}{r}1706187 \\
(11,3 \%)\end{array}$ & $\begin{array}{r}579289 \\
(9,2 \%)\end{array}$ & $\begin{array}{r}2010481 \\
(13,6 \%)\end{array}$ & $\begin{array}{r}1737675 \\
(13,0 \%)\end{array}$ & $\begin{array}{r}7543849 \\
(12,4 \%)\end{array}$ \\
\hline Mato Grosso do Sul & $\begin{array}{r}102385 \\
(0,9 \%)\end{array}$ & $\begin{array}{r}114209 \\
(0,8 \%)\end{array}$ & $\begin{array}{r}51852 \\
(0,8 \%)\end{array}$ & $\begin{array}{r}145329 \\
(1,0 \%)\end{array}$ & $\begin{array}{r}124968 \\
(0,9 \%)\end{array}$ & $\begin{array}{r}538743 \\
(0,9 \%)\end{array}$ \\
\hline Mato Grosso & $\begin{array}{r}100544 \\
(0,9 \%)\end{array}$ & $\begin{array}{r}126828 \\
(0,8 \%)\end{array}$ & $\begin{array}{r}65436 \\
(1,0 \%)\end{array}$ & $\begin{array}{r}155945 \\
(1,1 \%)\end{array}$ & $\begin{array}{r}133951 \\
(1,0 \%)\end{array}$ & $\begin{array}{r}582704 \\
(1,0 \%)\end{array}$ \\
\hline Pará & $\begin{array}{r}139571 \\
(1,3 \%)\end{array}$ & $\begin{array}{r}169757 \\
(1,1 \%)\end{array}$ & $\begin{array}{r}474762 \\
(7,5 \%)\end{array}$ & $\begin{array}{r}177876 \\
(1,2 \%)\end{array}$ & $\begin{array}{r}146685 \\
(1,1 \%)\end{array}$ & $\begin{array}{r}1108651 \\
(1,8 \%)\end{array}$ \\
\hline Paraíba & $\begin{array}{r}118027 \\
(1,1 \%)\end{array}$ & $\begin{array}{r}136836 \\
(0,9 \%)\end{array}$ & $\begin{array}{r}71338 \\
(1,1 \%)\end{array}$ & $\begin{array}{r}171154 \\
(1,2 \%)\end{array}$ & $\begin{array}{r}150349 \\
(1,1 \%)\end{array}$ & $\begin{array}{r}647704 \\
(1,1 \%)\end{array}$ \\
\hline Pernambuco & $\begin{array}{r}319926 \\
(2,9 \%)\end{array}$ & $\begin{array}{r}375292 \\
(2,5 \%)\end{array}$ & $\begin{array}{r}171792 \\
(2,7 \%)\end{array}$ & $\begin{array}{r}389204 \\
(2,6 \%)\end{array}$ & $\begin{array}{r}341332 \\
(2,6 \%)\end{array}$ & $\begin{array}{r}1597546 \\
(2,6 \%)\end{array}$ \\
\hline Piauí & $67449(0,6 \%)$ & $\begin{array}{r}85345 \\
(0,6 \%)\end{array}$ & $\begin{array}{r}20933 \\
(0,3 \%)\end{array}$ & $\begin{array}{r}101794 \\
(0,7 \%)\end{array}$ & $\begin{array}{r}81041 \\
(0,6 \%)\end{array}$ & $\begin{array}{r}356562 \\
(0,6 \%)\end{array}$ \\
\hline Paraná & $\begin{array}{r}937180 \\
(8,5 \%)\end{array}$ & $\begin{array}{r}1014908 \\
(6,7 \%)\end{array}$ & $\begin{array}{r}341779 \\
(5,4 \%)\end{array}$ & $\begin{array}{r}2079875 \\
(14,0 \%)\end{array}$ & $\begin{array}{r}2006117 \\
(15,1 \%)\end{array}$ & $\begin{array}{r}6379859 \\
(10,5 \%)\end{array}$ \\
\hline Rio de Janeiro & $\begin{array}{r}759621 \\
(6,9 \%)\end{array}$ & $\begin{array}{r}731021 \\
(4,8 \%)\end{array}$ & $\begin{array}{r}453539 \\
(7,2 \%)\end{array}$ & $\begin{array}{r}786956 \\
(5,3 \%)\end{array}$ & $\begin{array}{r}625983 \\
(4,7 \%)\end{array}$ & $\begin{array}{r}3357120 \\
(5,5 \%)\end{array}$ \\
\hline Rio Grande do Notte & $\begin{array}{r}118328 \\
(1,1 \%)\end{array}$ & $\begin{array}{r}132520 \\
(0,9 \%)\end{array}$ & $\begin{array}{r}63333 \\
(1,0 \%)\end{array}$ & $\begin{array}{r}144879 \\
(1,0 \%)\end{array}$ & $\begin{array}{r}131462 \\
(1,0 \%)\end{array}$ & $\begin{array}{r}590522 \\
(1,0 \%)\end{array}$ \\
\hline Rondônia & $52607(0,5 \%)$ & $\begin{array}{r}60542 \\
(0,4 \%)\end{array}$ & $\begin{array}{r}33246 \\
(0,5 \%)\end{array}$ & $\begin{array}{r}65841 \\
(0,4 \%)\end{array}$ & $\begin{array}{r}61309 \\
(0,5 \%)\end{array}$ & $\begin{array}{r}273545 \\
(0,5 \%)\end{array}$ \\
\hline Roraima & $9514(0,1 \%)$ & $\begin{array}{r}11800 \\
(0,1 \%)\end{array}$ & $3672(0,1 \%)$ & $\begin{array}{l}13982 \\
(0,1 \%)\end{array}$ & $\begin{array}{r}10735 \\
(0,1 \%)\end{array}$ & $\begin{array}{l}49703 \\
(0,1 \%)\end{array}$ \\
\hline Rio Grande do Sul & $\begin{array}{r}1017310 \\
(9,2 \%)\end{array}$ & $\begin{array}{r}3479644 \\
(23,0 \%)\end{array}$ & $\begin{array}{r}631857 \\
(10,0 \%)\end{array}$ & $\begin{array}{r}1278450 \\
(8,6 \%)\end{array}$ & $\begin{array}{r}1061088 \\
(8,0 \%)\end{array}$ & $\begin{array}{r}7468349 \\
(12,3 \%)\end{array}$ \\
\hline Santa Catarina & $\begin{array}{r}688746 \\
(6,2 \%)\end{array}$ & $\begin{array}{r}753805 \\
(5,0 \%)\end{array}$ & $\begin{array}{r}404447 \\
(6,4 \%)\end{array}$ & $\begin{array}{r}874824 \\
(5,9 \%)\end{array}$ & $\begin{array}{r}721300 \\
(5,4 \%)\end{array}$ & $\begin{array}{r}3443122 \\
(5,7 \%)\end{array}$ \\
\hline Sergipe & $76086(0,7 \%)$ & $\begin{array}{r}89043 \\
(0,6 \%)\end{array}$ & $\begin{array}{r}33003 \\
(0,5 \%)\end{array}$ & $\begin{array}{r}95429 \\
(0,6 \%)\end{array}$ & $\begin{array}{r}81264 \\
(0,6 \%)\end{array}$ & $\begin{array}{r}374825 \\
(0,6 \%)\end{array}$ \\
\hline São Paulo & $\begin{array}{c}3009788 \\
(27,1 \%)\end{array}$ & $\begin{array}{r}3297188 \\
(21,8 \%)\end{array}$ & $\begin{array}{r}1966197 \\
(31,1 \%)\end{array}$ & $\begin{array}{r}3712490 \\
(25,1 \%)\end{array}$ & $\begin{array}{r}3137298 \\
(23,6 \%)\end{array}$ & $\begin{array}{r}15122961 \\
(24,9 \%)\end{array}$ \\
\hline Tocantins & $37035(0,3 \%)$ & $\begin{array}{r}48581 \\
(0,3 \%) \\
\end{array}$ & $\begin{array}{r}11292 \\
(0,2 \%) \\
\end{array}$ & $\begin{array}{r}56857 \\
(0,4 \%) \\
\end{array}$ & $\begin{array}{r}48293 \\
(0,4 \%) \\
\end{array}$ & $\begin{array}{r}202058 \\
(0,3 \%) \\
\end{array}$ \\
\hline Total & $\begin{array}{r}11089115 \\
(100,0 \%) \\
\end{array}$ & $\begin{array}{r}15128576 \\
(100,0 \%)\end{array}$ & $\begin{array}{r}6328973 \\
(100,0 \%) \\
\end{array}$ & $\begin{array}{r}14808257 \\
(100,0 \%) \\
\end{array}$ & $\begin{array}{r}13320567 \\
(100,0 \%)\end{array}$ & $\begin{array}{r}60675488 \\
(100,0 \%)\end{array}$ \\
\hline
\end{tabular}

Fonte: Elaboração própria dos autores (2020).

Ao avaliar a dispensação de opioides quanto à origem do registro profissional do prescritor (Tabela 3), os médicos foram os principais prescritores. Fosfato de codeína foi o medicamento mais prescrito pelos médicos $(56,0 \%)$ e pelos dentistas $(85,6 \%)$, enquanto entre os profissionais da classe veterinária e entre os médicos intercambistas, o princípio ativo mais prescrito foi o cloridrato de tramadol, representando $96,7 \%$ e $44,9 \%$ dos opioides, respectivamente. 
Tabela 3. Porcentagem de opioides por ano, em função da origem do registro profissional do prescritor (Brasil, anos de 2014 a 2018).

\begin{tabular}{|c|c|c|c|c|c|c|}
\hline \multirow{3}{*}{ Registro do profissional prescritor } & \multicolumn{5}{|c|}{ Ano } & \multirow{2}{*}{ Total } \\
\hline & 2014 & 2015 & 2016 & 2017 & 2018 & \\
\hline & $\begin{array}{c}\mathrm{N} \\
(\%)\end{array}$ & $\begin{array}{c}\mathrm{N} \\
(\%)\end{array}$ & $\begin{array}{l}\mathrm{N} \\
(\%)\end{array}$ & $\begin{array}{c}\mathrm{N} \\
(\%)\end{array}$ & $\begin{array}{c}\mathrm{N} \\
(\%)\end{array}$ & $\begin{array}{c}\mathrm{N} \\
(\%)\end{array}$ \\
\hline $\mathrm{CRM}^{\mathrm{a}}$ & $\begin{array}{c}10611117 \\
(95,7 \%)\end{array}$ & $\begin{array}{c}14537699 \\
(96,1 \%)\end{array}$ & $\begin{array}{l}6050335 \\
(95,6 \%)\end{array}$ & $\begin{array}{c}13175518 \\
(89,0 \%)\end{array}$ & $\begin{array}{c}12162915 \\
(91,3 \%)\end{array}$ & $\begin{array}{c}56537584 \\
(93,2 \%)\end{array}$ \\
\hline $\mathrm{CRMV}^{\mathrm{b}}$ & $\begin{array}{l}188626 \\
(1,7 \%)\end{array}$ & $\begin{array}{c}222562 \\
(1,5 \%)\end{array}$ & $\begin{array}{l}87747 \\
(1,4 \%)\end{array}$ & $\begin{array}{c}1169390 \\
(7,9 \%)\end{array}$ & $\begin{array}{c}204239 \\
(1,5 \%)\end{array}$ & $\begin{array}{c}1872564 \\
(3,1 \%)\end{array}$ \\
\hline $\mathrm{CRO}^{\mathrm{c}}$ & $\begin{array}{l}248077 \\
(2,2 \%)\end{array}$ & $\begin{array}{c}291400 \\
(1,9 \%)\end{array}$ & $\begin{array}{l}150963 \\
(2,4 \%)\end{array}$ & $\begin{array}{c}367589 \\
(2,5 \%)\end{array}$ & $\begin{array}{c}860280 \\
(6,5 \%)\end{array}$ & $\begin{array}{c}1918309 \\
(3,2 \%)\end{array}$ \\
\hline $\mathrm{RMS}^{\mathrm{d}}$ & $\begin{array}{l}41295 \\
(0,4 \%)\end{array}$ & $\begin{array}{l}76915 \\
(0,5 \%)\end{array}$ & $\begin{array}{l}39928 \\
(0,6 \%)\end{array}$ & $\begin{array}{l}95760 \\
(0,6 \%)\end{array}$ & $\begin{array}{l}93133 \\
(0,7 \%)\end{array}$ & $\begin{array}{l}347031 \\
(0,6 \%)\end{array}$ \\
\hline Total & $\begin{array}{l}11089115 \\
(100,0 \%)\end{array}$ & $\begin{array}{l}15128576 \\
(100,0 \%)\end{array}$ & $\begin{array}{c}6328973 \\
(100,0 \%)\end{array}$ & $\begin{array}{l}14808257 \\
(100,0 \%)\end{array}$ & $\begin{array}{l}13320567 \\
(100,0 \%)\end{array}$ & $\begin{array}{c}60675488 \\
(100,0 \%)\end{array}$ \\
\hline
\end{tabular}

aCRM - Médico; bCRMV - Médico Veterinário; cCRO - Odontólogo; dRMS - Registro

Único do Ministério da Saúde (Médicos Intercambistas).

Fonte: Elaboração própria dos autores (2020)

\section{Discussão}

A avaliação de dados sobre a comercialização de medicações opioides no Brasil mostrou que a venda desses analgésicos entre os anos de 2009 e 2015 sofreu substancial aumento temporal (Krawczyk et al., 2018) no entanto, no período avaliado no presente estudo (2014 a 2018) não foi evidenciada tendência de aumento na venda desses fármacos, o que demonstra que aparentemente a prescrição desses medicamentos está sendo realizada de forma controlada e não há indícios para o desenvolvimento de uma epidemia por uso de opioides no Brasil, como a recentemente enfrentada em outros países.

Apesar de benéfica e bem documentada no controle da dor aguda e crônica, a ampliação do acesso a essas medicações pode trazer riscos inerentes às características das drogas, como a ocorrência de reações adversas, até o desenvolvimento de comportamentos, como o vício. Tal comportamento se torna um problema de saúde pública uma vez que os dependentes da medicação passam a tentar obter opioides de forma ilícita (Calônego, 2020; Gomes et al., 2020).

Diferente do observado nos Estados Unidos, principalmente durante os anos de 2016 e 2017, que passou pelo que foi denominado de Crise do Opioide (Meyer et al., 2020), com ampla discussão ética sobre a indicação terapêutica desta classe de medicamento; no Brasil não há registros de intoxicações e overdose, pois o consumo de derivados de opioides é considerado baixo (Pereira et al., 2017).

Conforme determinação da Secretaria de Vigilância em Saúde, opioides somente são dispensados a partir de prescrição médica específica associada à retenção da receita, o que pode contribuir para o aumento da segurança em saúde pública quanto ao uso desses analgésicos no país (Pereira et al., 2017; Calônego, 2020). Além disso, já foi identificada entre a classe médica a existência de receio quanto a prescrição de opioides, em virtude da falta de conhecimento da farmacologia e da legislação envolvendo esse tipo de medição (Calônego, 2020).

No período avaliado, o ano de 2016 mostrou um declínio importante na comercialização de analgésicos de ação central. Apesar de no presente estudo não ser possível identificar a existência de eventual falha no sistema de informação utilizado, pode-se inferir que este achado se relaciona à crise político-econômica que acometeu o Brasil no ano de 2016 quando a economia desacelerou, diminuindo os investimentos públicos em saúde, além do aumento na taxa de desemprego reduzindo e o poder de compra dos brasileiros (Duminelli et al., 2016; Paula; Pires, 2017).

Krawczyk et al (2018) verificaram que entre os anos de 2009 e 2015 a oxicodona foi o produto de opioide com maior aumento relativo em prescrição no Brasil e em contraponto, o presente estudo apontou que a medicação com maior aumento relativo na dispensação foi a codeína, prescrita por médicos, o que pode ser compreendido pelo fato dessa medicação estar incluída na lista de medicamentos essenciais no tratamento da dor oncológica no Brasil, além de ser um medicamento de 
primeira escolha para tratamento de dores agudas e crônicas, principalmente as de origem traumática ou oncológica (Kahan et al., 2011; Brasil, 2006; Salamonde et al., 2006).

Em contraste, os veterinários prescreveram mais o cloridrato de tramadol. Embora os dados do presente estudo não contenham informações para explicar as razões dessa diferença, infere-se que a existência de uma formulação a base de cloridrato de tramadol específica na indústria veterinária pôde popularizar o uso deste, uma vez que o mesmo é amplamente utilizado no manejo da dor em animais, principalmente após procedimentos cirúrgicos (Sudheer et al., 2007; Marangoni et al., 2014; Evangelista et al., 2014).

Opioides não estão entre as principais escolhas analgésicas nas práticas odontológicas, exceto quando associados com drogas de ação periférica, sejam analgésicos ou anti-inflamatórios, fato decorrente da terapêutica em odontologia apresentar algumas particularidades em relação a característica da dor, usualmente inflamatória e aguda, de severidade moderada e curta duração na maioria das situações clínicas, além disso sua prescrição excessiva pode ser um gatilho para a ocorrência de farmacodependência entre os pacientes (Priest et al., 2020; Wilkem; Abreu, 2017). O menor número de prescrição de opioides por dentistas brasileiros, pôde ser observado no presente estudo, uma vez que a prescrição de opioides por essa categoria profissional representou apenas 3,2\% do total de medicamentos prescritos.

A prescrição de opioides ocorreu em todos os estados brasileiros, sendo o fosfato de codeína o mais prescrito. Embora as taxas de prescrição tenham aumentado ao longo dos anos considerando-se a comparação com os dados encontrados por Krawczyk et al. (2018) no período de 2009 a 2015, no presente estudo não foi observada tendência de aumento no consumo de opioides o que nos permite inferir que aparentemente não há indícios que o país possa enfrentar crise envolvendo a dependência farmacológica quanto ao uso de opioides.

Diante dos riscos envolvendo o uso desta classe de analgésicos torna-se necessário monitorar a venda desses medicamentos de forma sistemática e contínua mesmo no cenário identificado entre 2009 e 2015 (Krawczyk et al, 2018) e atualizado no presente estudo até 2018 em que as taxas de uso de opioides prescritos no Brasil permanecem muito aquém das observadas na América do Norte. Neste contexto, faz-se importante ações que garantam o adequado monitoramento da venda desses medicamentos, principalmente no que se refere à comercialização de opioides da indústria veterinária, o uso adequado dos opioides prescritos além da manutenção das precauções em nível de política e de sistema para proteger a saúde pública e prevenir o desenvolvimento de outra epidemia devastadora no país.

Este estudo apresenta como limitação, o fato de trabalhar com informações disponíveis em banco de dados, o que não permitiu uma análise do uso indevido dos opioides, por exemplo. Os dados também não permitiram diferenciar as prescrições realizadas por profissionais da saúde das prescrições realizadas por médico veterinários com relação a dose, posologia e duração de tratamento. Tais informações nos ajudariam a compreender o perfil de prescrição desta classe de analgésicos, mas de todo modo, face à necessidade de melhorar o controle da dor e de compreender os benefícios e riscos do uso de opioides é fundamental acompanhar a tendência na utilização de opioides e os dados do presente estudo contribuem nesse sentido.

\section{Conclusão}

A prescrição de opioides ocorreu em todos os estados brasileiros, sendo o fosfato de codeína o mais prescrito. Embora não tenha sido observada tendência de aumento no consumo, o monitoramento da venda desses medicamentos deve ser intensificado de forma que os pacientes consigam acessá-los realmente quando necessário, limitando a possibilidade de uso indevido considerando os riscos envolvidos. Ressalta-se que ainda há a necessidade de aumentar o conhecimento dos prescritores sobre ferramentas farmacológicas alternativas não opióides e, portanto, com menos potencial abusivo. 


\section{Referências}

Brasil. (2012). Uso racional de medicamentos: temas selecionados. Série A. Normas e Manuais Técnicos.

Brasil (2006). Assistência farmacêutica na atenção básica: instruções técnicas para sua organização. Série A. Normas e Manuais Técnicos, 2 Ed.

Calônego, M. A. M. (2020) Dificuldades sociais, legais e burocráticas para prescrição de opioides [tese]. Botucatu (SP): Universidade Estadual Paulista "Júlio de Mesquita Filho.

Coluzzi, F., Taylor, R., Jr, Pergolizzi, J. V., Jr, Mattia, C., \& Raffa, R. B. (2016). Good clinical practice guide for opioids in pain management: the three Ts titration (trial), tweaking (tailoring), transition (tapering). Brazilian journal of anesthesiology (Elsevier), 66(3), 310-317. https://doi.org/10.1016/j.bjane.2014.09.005

Duminelli, M. V., Redivo, T. S., \& Yamaguchi. C. K. (2016). Perspectiva da crise econômica e política no Brasil: reflexos para o desenvolvimento da economia com foco no setor do comércio. Revista Contribuciones a las Ciencias Sociales. 1(12): 01-04.

Evangelista, M. C., Silva, R. A., Cardozo, L. B., Kahvegian, M. A., Rossetto, T. C., Matera, J. M., \& Fantoni, D. T. (2014). Comparison of preoperative tramadol and pethidine on postoperative pain in cats undergoing ovariohysterectomy. BMC veterinary research, 10, 252. https://doi.org/10.1186/s12917-014$0252-1$

Gomes, T., Tadrous, M., Mamdani, M. M., Paterson, J. M., \& Juurlink, D. N. (2018). The Burden of Opioid-Related Mortality in the United States. JAMA network open, 1(2), e180217. https://doi.org/10.1001/jamanetworkopen.2018.0217

Gomes, V. P., Silva, M. T., \& Galvão, T. F. (2017). Prevalence of medicine use among Brazilian adults: a systematic review. Prevalência do consumo de medicamentos em adultos brasileiros: uma revisão sistemática. Ciencia \& saude coletiva, 22(8), 2615-2626. https://doi.org/10.1590/141381232017228.29412016

Kahan, M., Mailis-Gagnon, A., \& Tunks, E. (2011). Canadian guideline for safe and effective use of opioids for chronic non-cancer pain: implications for pain physicians. Pain research \& management, 16(3), 157-158. https://doi.org/10.1155/2011/434298

Krawczyk, N., Greene, M. C., Zorzanelli, R., \& Bastos, F. I. (2018). Rising Trends of Prescription Opioid Sales in Contemporary Brazil, 2009-2015. American journal of public health, 108(5), 666-668. https://doi.org/10.2105/AJPH.2018.304341

Marangoni. J.M., Franca, L.G., \& Palazzo, E.L. (2014). Uso do Tramadol em Cães e Gatos - Revisão. Nucleus. $11(2)$ : 1.

Meyboom, R. H., Lindquist, M., \& Egberts, A. C. (2000). An ABC of drug-related problems. Drug safety, 22(6), 415-423. https://doi.org/10.2165/00002018200022060-00001

Meyer, A., LeClair, C., \& McDonald, J. V. (2020). Prescription Opioid Prescribing in Western Europe and the United States. Rhode Island medical journal (2013), 103(2), 45-48.

Nascimento, D. C. H., \& Sakata, R. K. (2011). Dependência de opioide em pacientes com dor crônica. Revista dor, 12, 160-165.

Ngoundo-Mbongue, T. B., Niezborala, M., Sulem, P., Briant-Vincens, D., Bancarel, Y., Jansou, P., Chastan, E., Montastruc, J. L., \& Lapeyre-Mestre, M. (2005). Psychoactive drug consumption: performance-enhancing behaviour and pharmacodependence in workers. Pharmacoepidemiology and drug safety, 14(2), 81-89. https://doi.org/10.1002/pds.1006

Paula, L.F., \& Pires, M. (2017). Crise e perspectivas para a economia brasileira. Estudos avançados. 31 (89): $125-144$.

Pereira, M. de M., Andrade, L. de P., \& Takitane, J. (2017). Evolução do uso abusivo de derivados de ópio. Saúde Ética \& Justiça, 21 (1), 12-17. https://doi.org/10.11606/issn.2317-2770.v21i1p12-17

Priest, C. R., Kenney, B. C., Brummett, C. M., Waljee, J. F., Englesbe, M. J., \& Nalliah, R. P. (2020). Increased opioid prescription fills after dental procedures performed before weekends and holidays. Journal of the American Dental Association (1939), 151(6), 388-398.e1. https://doi.org/10.1016/j.adaj.2020.03.014

Rudd, R. A., Seth, P., David, F., \& Scholl, L. (2016). Increases in Drug and Opioid-Involved Overdose Deaths - United States, 2010-2015. MMWR. Morbidity and mortality weekly report, 65(50-51), 1445-1452. https://doi.org/10.15585/mmwr.mm655051e1

Salamonde, G. L., Verçosa, N., Barrucand, L., \& Costa, A. F. (2006). Análise clínica e terapêutica dos pacientes oncológicos atendidos no programa de dor e cuidados paliativos do Hospital Universitário Clementino Fraga Filho no ano de 2003 [Clinical and therapeutic analysis of oncology patients treated at the pain and palliative care program of the Hospital Universitário Clementino Fraga Filho in 2003.]. Revista brasileira de anestesiologia, 56(6), 602-618. https://doi.org/10.1590/s0034-70942006000600005

Sudheer, P. S., Logan, S. W., Terblanche, C., Ateleanu, B., \& Hall, J. E. (2007). Comparison of the analgesic efficacy and respiratory effects of morphine, tramadol and codeine after craniotomy. Anaesthesia, 62(6), 555-560. https://doi.org/10.1111/j.1365-2044.2007.05038.x

Volkow, N. D., McLellan, T. A., Cotto, J. H., Karithanom, M., \& Weiss, S. R. (2011). Characteristics of opioid prescriptions in 2009. JAMA, 305(13), 12991301. https://doi.org/10.1001/jama.2011.401

Wilken, I. S., \& de Abreu, M. H. N. G. (2017). Utilização de analgésicos opioides após exodontia de terceiros molares: uma revisão da literatura científica. Arquivos Em Odontologia, 53. https://periodicos.ufmg.br/index.php/arquivosemodontologia/article/view/3728 